\title{
Extensively drug-resistant Klebsiella pneumoniae ST307 outbreak, north-eastern Germany, June to October 2019
}

Sebastian Haller ${ }^{1}$, Rolf Kramer ${ }^{1}$, Karsten Becker ${ }^{5}$, Jürgen A Bohnert ${ }^{5}$, Tim Eckmanns ${ }^{1}$, Jörg B Hans ${ }^{6}$, Jane Hecht ${ }^{1}$, Claus-Dieter Heidecke $^{5}$, Nils-Olaf Hübner ${ }^{5}$, Axel Kramer 5 , Kathleen Klaper ${ }^{2}$, Martina Littmann 4 , Lennart Marlinghaus ${ }^{6}$, Bernd Neumann²,

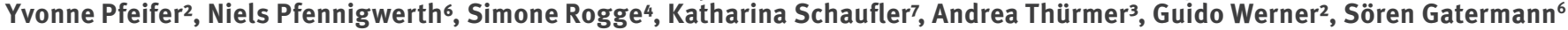

1. Robert Koch Institute, Department for Infectious Disease Epidemiology, Berlin, Germany

2. Robert Koch Institute, Division of Nosocomial Pathogens and Antibiotic Resistance, Wernigerode, Germany

3. Robert Koch Institute, Genome Sequencing Unit, Berlin, Germany

4. Regional Public Health Authority Mecklenburg-Western Pomerania, Rostock, Germany

5. University Medicine Greifswald, Greifswald, Germany

6. National Reference Centre for multidrug-resistant Gram-negative bacteria, Ruhr University Bochum, Bochum, Germany

7. Institute of Pharmacy, University of Greifswald, Greifswald, Germany

Correspondence: Sebastian Haller (HallerS@rki.de)

Citation style for this article:

Haller Sebastian, Kramer Rolf, Becker Karsten, Bohnert Jürgen A, Eckmanns Tim, Hans Jörg B, Hecht Jane, Heidecke Claus-Dieter, Hübner Nils-Olaf, Kramer Axel, Klaper Kathleen, Littmann Martina, Marlinghaus Lennart, Neumann Bernd, Pfeifer Yvonne, Pfennigwerth Niels, Rogge Simone, Schaufler Katharina, Thürmer

Andrea, Werner Guido, Gatermann Sören. Extensively drug-resistant Klebsiella pneumoniae ST307 outbreak, north-eastern Germany, June to October 2019 . Euro Andrea, Werner Guido, Gatermann Sören. Extensively drug-resistant Klebsiella pneumoniae ST307
Surveill. 2019;24(50):pii=1900734. https://doi.org/10.2807/1560-7917.ES.2019.24.50.1900734

Article submitted on 03 Dec 2019 / accepted on 12 Dec 2019 / published on 12 Dec 2019

From June to October 2019, 17 patients (six infected, 11 colonised) with an extensively drug-resistant (XDR) Klebsiella pneumoniae strain were notified from four Western Pomerania medical facilities. The XDR K. pneumoniae produced carbapenemases NDM-1 and OXA-48, and was only susceptible to chloramphenicol, tigecycline and cefiderocol. Synergistic activity was observed for the combination of aztreonam plus ceftazidime-avibactam. Genomic analyses showed all isolates belonged to $K$. pneumoniae sequence type 307. Control measures and further investigations are ongoing.

Between June and October 2019, a university hospital, two other hospitals and a rehabilitation clinic in northeastern Germany (Western Pomerania, Greifswald) were affected by an outbreak of extensively drug-resistant (XDR) Klebsiella pneumoniae. A total of 17 patients were infected or colonised. The aim of this short article is to provide detailed information on the epidemiological and microbiological results of this outbreak investigation, and thereby facilitate comparison with similar outbreaks of this emerging Klebsiella strain on international level.

\section{Outbreak detection and investigation}

Following a routine microbiological screening of tracheal secretion on an intensive care unit (ICU) at a university hospital, the first patient with XDR K. pneumoniae was detected on 25 June in calendar week 26. The patient was placed in a single room and enhanced barrier precautions were put in place. Contact patients were screened and an admission and exit screening for colonisation with Gram-negative bacteria was performed on the affected ICU in the university hospital. Despite extended precautions, further cases were detected on six wards at the university hospital throughout the following weeks. Under the impression of an ongoing outbreak, intensified measures such as screening, environmental testing and cohorting of cases to a special isolation unit were implemented. In the meantime, patients with XDR K. pneumoniae were detected in a primary-hospital, secondary-hospital and rehabilitation clinic in the region.

All isolates from all affected medical facilities were sent to the National Reference Centre (NRC) for multidrug-resistant (MDR) Gram-negative bacteria in Bochum. There the microbiological and molecular analyses of corresponding isolates revealed the production of two carbapenemases, NDM-1 and OXA-48, and colistin resistance.

A case was defined as a person carrying the K. pneumoniae outbreak clone as confirmed by molecular typing AND having an epidemiological link to the outbreak.

Cases and outbreak information were reported to the responsible health authorities. Extensive drug resistance, a high proportion of infections as well as challenges in outbreak control led to involvement of the national public health services (in calendar week 38 ).

Of the 17 cases (Figure 1), two were female and 15 were male, with a median age 70 years (range: $50-84$ years). Six cases presented clinical symptoms (sepsis, pneumonia, urinary tract infection), 11 cases were colonised; most had severe underlying diseases. Of the 17 cases, 10 were diagnosed in the university hospital, four in a primary hospital, two in the rehabilitation 


\section{FIGURE 1}

Number of Klebsiella pneumoniae ST307 outbreak cases according to date of first detection per calendar week, north-eastern Germany, June-October 2019

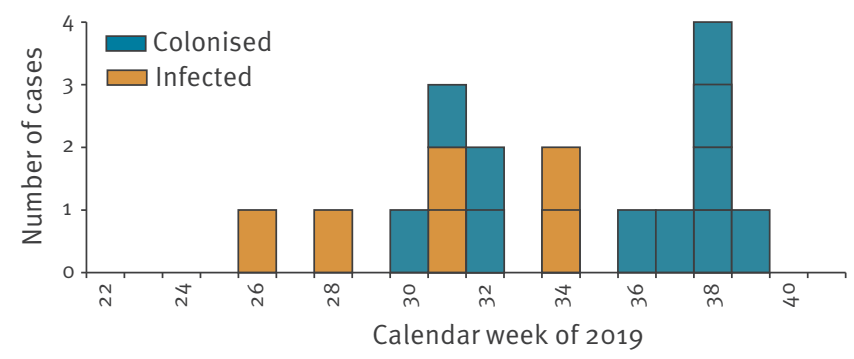

clinic and one in a secondary hospital (Figure 2). All but one case had been exposed to treatment in the university hospital before the first detection of the outbreak clone. The only case not exposed at the university hospital shared the same room with another case in the rehabilitation clinic (Figure 2). By 15 October 2019, six cases had died apparently from underlying diseases; nonetheless, causal links with the outbreak clone are under further investigation.

\section{Microbiological and molecular analyses}

Initial antibiotic susceptibility testing (AST) of 10 isolates using semi-automated VITEK 2 (Biomerieux, Nürtingen, Germany) analysis and 96-well plate broth microdilution (Merlin, Bornheim-Hersel, Germany) revealed resistance to a wide range of antibiotics. Testing for common carbapenemase gene families indicated presence of bla ${ }_{\text {OXA-48-like }}$ and bla ${ }_{\text {NDM }}$ in the isolates. Furthermore, isolates showed resistance against aztreonam and ceftazidime-avibactam. However, to assess therapeutic options an Etest synergy assay (bioMérieux, Nürtingen, Germany) with ceftazidime-avibactam and aztreonam was used, with results suggesting a synergistic activity. An extended AST showed extensive drug-resistance with susceptibility only to chloramphenicol, tigecycline and cefiderocol, the latter of which is not yet approved for use in European Union/ European Economic Area (EU/EEA) countries.

For early discrimination of outbreak and non-outbreak isolates, a loop-mediated isothermal amplification (LAMP) assay (eazyplex SuperBug CRE, AmplexDiagnostics, Gars, Germany) for rapid detection of the carbapenemase genes was included in the university hospital laboratory diagnostic procedure.

The NRC performed molecular typing by Xbalmacrorestriction analyses in PFGE. Following the criteria of Tenover et al. [1], analyses revealed that all isolates of the 17 cases belonged to the same outbreak strain (Supplementary Figure S1).

Thirteen isolates from the first eleven patients were available for whole-genome sequencing (WGS) using the MiSeq System (Illumina, San Diego, United
States). Sequence analyses and typing were done using Kleborate tool and other established methods [2-6]. Core genome (cg)MLST analyses with the Ridom SeqSphere $^{+}$software version 6.0.0 confirmed PFGE results, showing a maximum of 12 allelic differences between isolates (Figure 3). In silico typing revealed that all isolates belonged to sequence type (ST)307 and cgMLST complex type CT3459, with the capsule type K2 (wzi-173, wzC-939). Sequence analyses apart from carbapenemase encoding genes revealed a broad spectrum of further resistance genes, e.g. bla стх-мqnrS1 (Table). There was no evidence for the presence of the transmissible colistin resistance gene mcr-1 or related variants. However, amino acid substitutions were identified in intrinsic two component systems, PmrA/PmrB and PhoP/PhoQ, which have previously been shown to mediate colistin resistance [7].

\section{Outbreak control measures}

Healthcare facilities, general practitioners and regional medical laboratories of the region were informed about the outbreak by public health authorities and the regional multidrug-resistant organisms (MDRO)network on 24.09.2019.

In calendar week 38, an extended outbreak meeting with the federal, state and regional public health authorities, the affected facilities and the regional MDRO-network was organised to harmonise case search, follow-up of cases and hygiene measures. From calendar week 39 onwards, all institutions with more than one case performed one-time screening of all patients, prevalence screening, on all wards to make sure that existence of undetected cases was unlikely. In addition, a weekly screening was performed for 6 weeks starting at calendar week 40 on all wards where a case had stayed overnight. A notification of discharged patients that were hospitalised during the outbreak when transmission was not yet controlled, was discussed, but not introduced. Instead, all hospitals in the region were asked to perform an admission screening on patients that were exposed to one of the outbreak facilities from June 2019 onwards. The last transmission was detected in calendar week 39.

An overview about this outbreak with a brief description of the pathogen was published in the weekly German national bulletin on 2 October 2019, and information was internationally shared via the European Centre for Disease Prevention and Control's (ECDC) Early Warning and Response System (EWRS) and Epidemic Intelligence Information System (EPIS) and the World Health Organization's (WHO) Global Antimicrobial Resistance Surveillance System-Emerging Antimicrobial Resistance Reporting (GLASS-EAR) [9].

\section{Discussion}

This is the first reported nosocomial outbreak of XDR K. pneumoniae ST307 with NDM-1, OXA-48 and CTX-M-15 in Germany. Despite established hygiene precautions, a university hospital, two other hospitals 


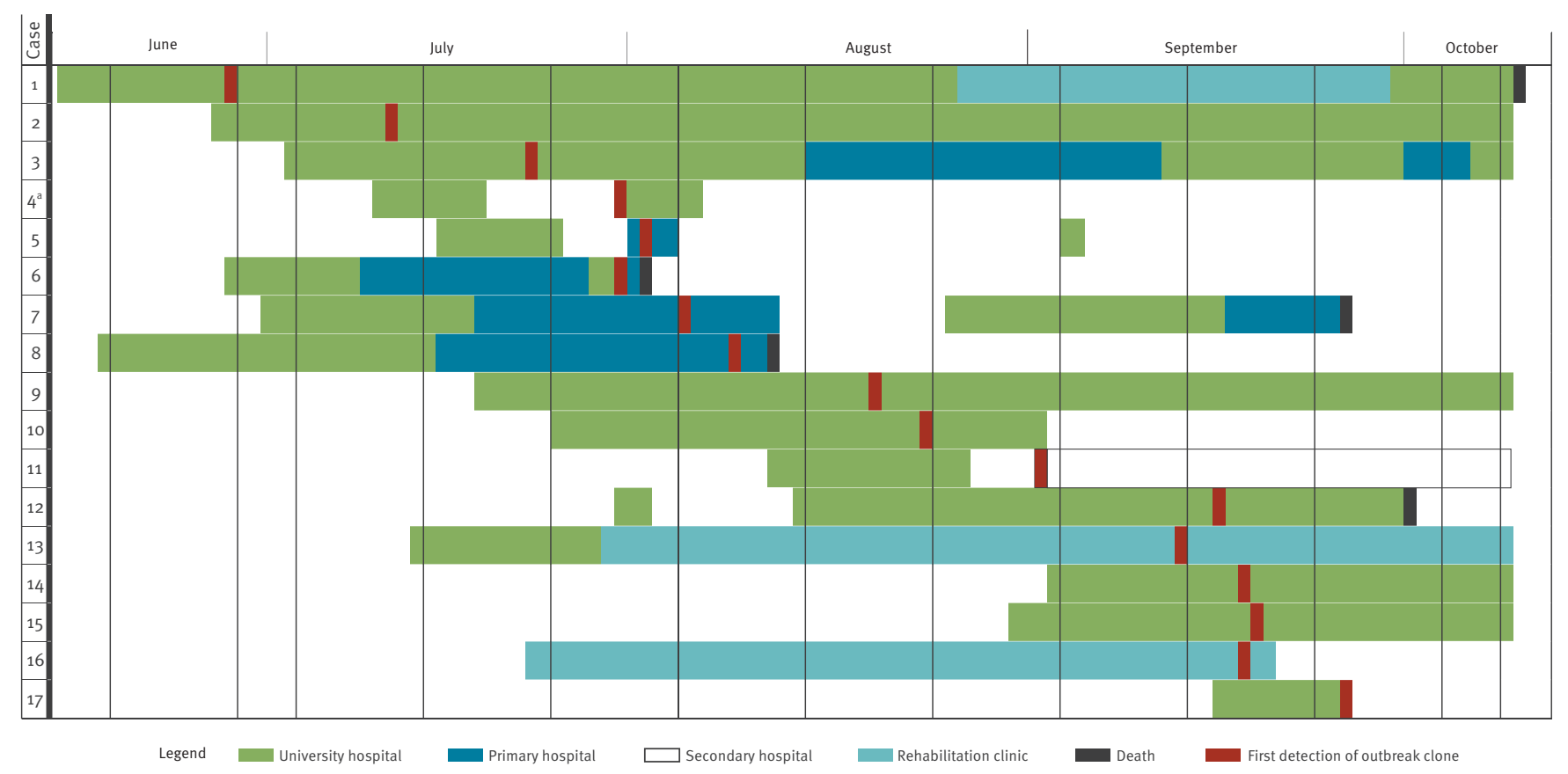

Time intervals of 10 days between vertical lines. Dates of detection reported as known by the Robert Koch Institute. Case 1 was hospitalised on 7 June 2019 and died on 15 October 2019. A total of six cases died, of which Case 4 died after discharge (adate not specified). The first detection of the outbreak strain was on 25 June 2019 (Case 1) and Case 17 was detected on 26 September 2019.

and a rehabilitation clinic in north-eastern Germany were affected. This may suggest that the outbreak clone is highly adapted to the hospital environment. Microbiological analyses of the outbreak strain are ongoing, preliminary results of susceptibility testing to disinfectants does not suggest increased disinfectant resistance.

The outbreak seemed controlled until week 44 as no new transmissions occurred. In calendar week 44, however, Case 18 was detected through admission screening in the university hospital. This case had been hospitalised during July and September on wards of the university hospital with other cases and had not been screened during these stays. Identification of this case indicates that further unknown cases may exist. This highlights the need for close information exchange between referral hospitals and for screening of patients that were exposed to one of the outbreak facilities from June 2019 onwards. A new case was also detected through rectal screening in week 45 . This case, Case 19, stayed on the ward where current cases are being cohorted and a recent transmission event seems most likely. Again an extended outbreak meeting with all stakeholders was organised in week 46 and barrier precautions were reinforced. Currently data collection of these two recent cases as well as sequencing of the isolates are being conducted. PFGE analysis of the new isolates shows that these belonged to the outbreak strain. As of calendar week 49, there have been no further cases detected.

The mode of transmission is still under investigation, but person-to-person transmission is most likely the relevant route. In this context, the following two steps are especially valuable: ensuring intensified isolation for cases and intensified case searching through systematic extended screening immediately when an outbreak is suspected, to avoid further unrecognised cases. Other outbreaks with similar pathogens have shown that systematic rectal screening is crucial to identifying colonised patients [10]. Early in-depth analysis of the molecular and phenotypic strain features supports decision making processes for treatment and outbreak control.

The first case (index patient) presented no typical risk factors for $K$. pneumoniae infection such as a recent hospital stay or recent travel, and is therefore unlikely to be the primary case that brought the outbreak strain into the university hospital. As exemplified by the detection of Case 18, there may have been undetected cases, especially during the early phase of the outbreak. All but one case (of the 19 cases) had a history of hospitalisation in the university hospital, which is the only tertiary hospital in the affected region. Transmissions within the other institutions involved cannot be ruled out. Cooperation between institutions and public health authorities should be intensified, e.g. 


\section{FIGURE 3}

Minimum spanning tree of 13 XDR Klebsiella pneumoniae ST307/CT3459 isolates of the outbreak based on allelic profiles, north-eastern Germany, June-October 2019
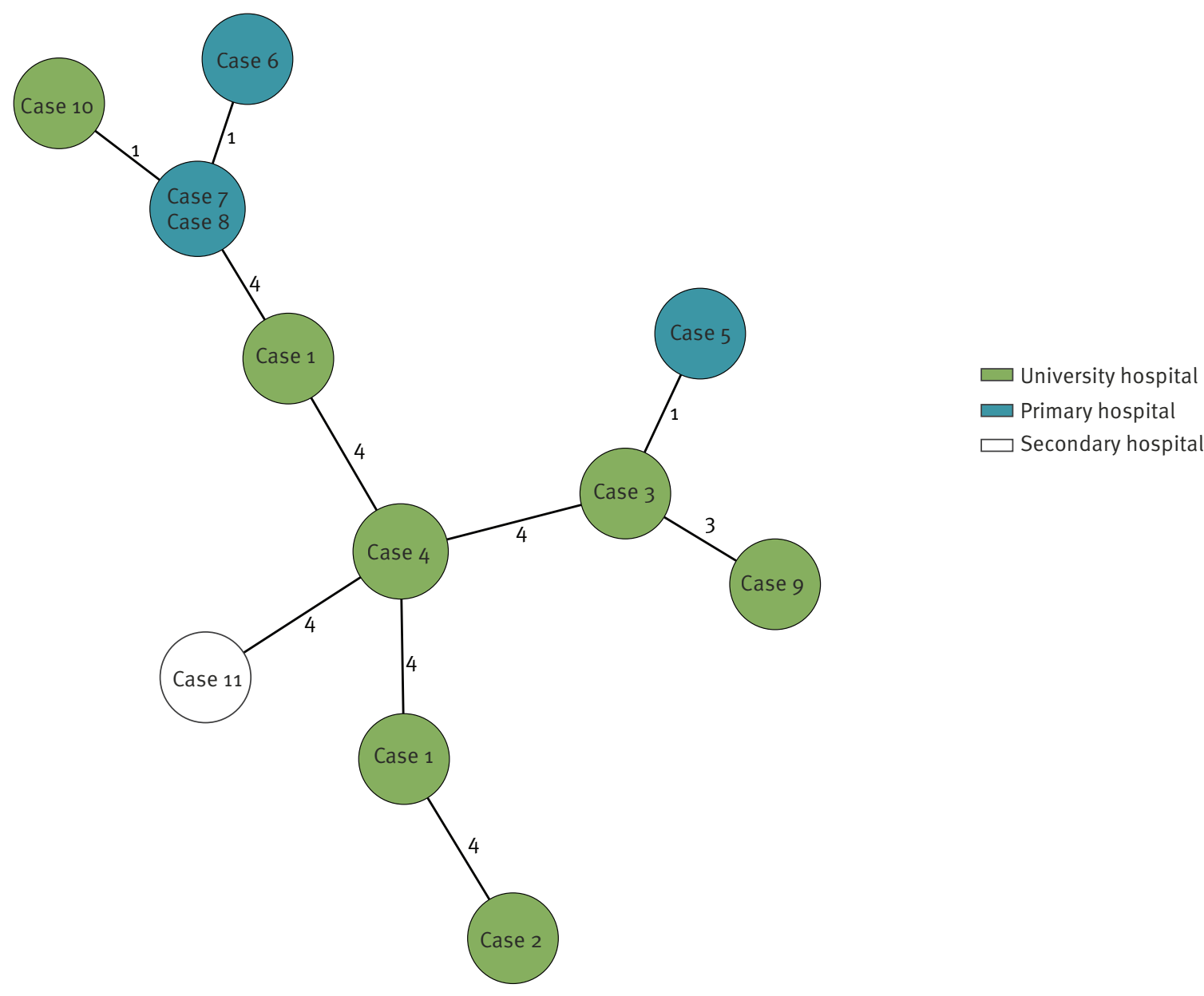

For each isolate, an allelic profile was determined by cgMLST analysis using the Ridom SeqSphere ${ }^{+}$software version 6.0.0. These profiles were used to determine both the complex type CT3459 and the genetic distances between isolates. Results visualise relatedness of isolates on core-genome scale as minimum spanning tree (MST). The MST was calculated with the option 'pairwise ignore missing values'. Each resulting circle represents a distinct allelic profile. Cases from which the isolates were collected are shown for each allelic profile. Two

isolates from Case 4 showed identical allelic profiles and were grouped together in a single circle (see also Case 7 and Case 8). Numbers on connecting lines represent allele differences between allelic profiles. Colours of circles indicate for each case the hospital where the outbreak strain was detected for the first time.

by fostering close, local collaboration between health facilities, health departments, laboratories and further stakeholders in MDRO-networks. This has already been proven to be successful for controlling outbreaks with other XDR pathogens [11].

The emergence of XDR $K$. pneumoniae dramatically limits treatment options. Only a few outbreaks of $K$. pneumoniae with the carbapenemase combination of NDM-1 and OXA-48 have been described $[12,13]$. Furthermore, presence of both carbapenemases NDM-1 and OXA-48 were identified in other K. pneumoniae sequence types $[14,15]$, but rarely in ST307 [16]. However, CTX-M-15-associated K. pneumoniae ST307 with OXA-48 and colistin resistance was reported in a Serbian hospital in 2015 [7]. Wyres et al. [17] recently described CTX-M-15-associated $K$. pneumoniae ST307 as a highly successful, globally emerging lineage with remarkable level of plasmid conservation.

Clinical and laboratory staff need to increase vigilance in order to improve early detection of XDR outbreaks. Early extensive screening and a high level of isolation precautions are needed to avoid further spread of these pathogens. As consequence of this outbreak, the detection of an XDR enterobacterial strain in an area with low endemicity emphasizes the need for increased awareness and risk mitigation measures to avoid transmission events. 
TABLE

Resistance genes detected in the Klebsiella pneumoniae ST307 outbreak strain using the Kleborate tool, north-eastern Germany, June-October 2019

\begin{tabular}{|c|c|}
\hline Affected antibiotic class & Detected genes/gene cluster \\
\hline \multirow{5}{*}{ Beta-lactams } & bla ${ }_{\mathrm{NDM}-1}$ \\
\hline & $b l a_{\text {OXA-48 }}$ \\
\hline & bla $a_{\mathrm{CTX}-\mathrm{M}-15}$ \\
\hline & $b l a_{\mathrm{TEM}-1 \mathrm{~B}}$ \\
\hline & bla $a_{\text {SHV-28-like }}$ \\
\hline \multirow{6}{*}{ Aminoglycosides } & Aac(3)-Ila-like \\
\hline & $a p h(3 ")-1 b$ \\
\hline & aph(3')-la-like \\
\hline & $\operatorname{aph}\left(3^{\circ}\right)-V I$ \\
\hline & Aph(6)-Id \\
\hline & $\operatorname{arm} A$ \\
\hline \multirow{5}{*}{ Quinolones } & oqxA-like \\
\hline & oqxB-like \\
\hline & $q n r S_{1}$ \\
\hline & gyrA 83 \\
\hline & parC 80 \\
\hline \multirow{3}{*}{ Macrolides } & mph(A)-like \\
\hline & $m p h(E)$ \\
\hline & $m s r(E)$ \\
\hline \multirow{2}{*}{ Sulphonamides } & sul1 \\
\hline & sul2 \\
\hline Fosfomycin & fosA-like \\
\hline Trimethoprim & $d f r A_{5}$ \\
\hline
\end{tabular}

\section{Acknowledgements}

We thank Sybille Müller-Bertling and Kirstin Ganske (MF2) and Anke Albrecht, Susanne Friedrich, Nadine Frey, Anja Kaminski and Julia Klessig (NRC) for excellent technical assistance. Special thanks to Stephan Fuchs for his support in NGS data QC and data transfer.

Funding statement: None

\section{Conflict of interest}

None declared.

\section{Authors' contributions}

SH, TE, JH, NOH, AK, ML and SR were part of the outbreak investigation team and conducted the epidemiological investigation. In addition, $\mathrm{NOH}$ and $\mathrm{AK}$ sent the initial alerts. $\mathrm{NOH}$, $A K, K B, J A B, C D H, M L$ and SR contributed to the investigations at the local level. ML and SR coordinated the investigation on the state level. SH, TE, GW and SG coordinated the investigation at the national level. GW and SG coordinated the typing investigations together with $\mathrm{KB}, \mathrm{KS}, \mathrm{JBH}, \mathrm{LM}, \mathrm{BN}$, KK, YP and AT. LM and NP performed AST, PCR-based screening and PFGE analysis. JBH, BN, KK, YP, GW and SG analysed WGS data. SH, RK and JH drafted the manuscript together with SG and TE. All authors contributed to the revision of the draft manuscript and approved the final version.

\section{References}

1. Tenover FC, Arbeit RD, Goering RV, Mickelsen PA, Murray BE, Persing DH, et al. Interpreting chromosomal DNA restriction patterns produced by pulsed-field gel electrophoresis: criteria for bacterial strain typing. J Clin Microbiol. 1995;33(9):2233-9. PMID: 7494007

2. Richter M, Rosselló-Móra R, Oliver Glöckner F, Peplies J. JSpeciesWS: a web server for prokaryotic species circumscription based on pairwise genome comparison. Bioinformatics. 2016;32(6):929-31. https://doi.org/10.1093/ bioinformatics/btv681 PMID: 26576653

3. Jolley KA, Maiden MC. BIGSdb: Scalable analysis of bacterial genome variation at the population level. BMC Bioinformatics. 2010;11(1):595. https://doi.org/10.1186/1471-2105-11-595 PMID: 21143983

4. Wick RR, Heinz E, Holt KE, Wyres KL. Kaptive Web: UserFriendly Capsule and Lipopolysaccharide Serotype Prediction for Klebsiella Genomes. J Clin Microbiol. 2018;56(6):e00197-18. https://doi.org/10.1128/JCM.00197-18 PMID: 29618504

5. Wyres KL, Wick RR, Gorrie C, Jenney A, Follador R, Thomson $N R$, et al. Identification of Klebsiella capsule synthesis loci from whole genome data. Microb Genom. 2016;2(12):e000102. https://doi.org/10.1099/mgen.0.000102 PMID: 28348840

6. Zankari E, Hasman H, Cosentino S, Vestergaard M, Rasmussen $\mathrm{S}$, Lund $\mathrm{O}$, et al. Identification of acquired antimicrobial resistance genes. J Antimicrob Chemother. 2012;67(11):2640-4. https://doi.org/10.1093/jac/dks261 PMID: 22782487

7. Novović K, Trudić A, Brkić S, Vasiljević Z, Kojić M, Medić D, et al. Molecular Epidemiology of Colistin-Resistant, Carbapenemase-Producing Klebsiella pneumoniae in Serbia from 2013 to 2016. Antimicrob Agents Chemother. 2017;61(5):e02550-16. https://doi.org/10.1128/AAC.02550-16 PMID: 28242665

8. European Centre for Disease Prevention and Control (ECDC). Outbreak of carbapenemase-producing (NDM-1 and OXA48) and colistin-resistant Klebsiella pneumoniae ST307, north-east Germany, 2019. Stockholm: ECDC; 2019. Available from: https://www.ecdc.europa.eu/en/publications-data/ outbreak-Klebsiella-pneumoniae-Germany

9. Robert Koch Institute (RKI). Information zum Auftreten von Klebsiella pneumoniae mit OXA-48 und NDM-1 Carbapenemasenund Colistinresistenz in MecklenburgVorpommern. [Information on the occurrence of Klebsiella pneumoniae with OXA-48 and NDM-1 carbapenemase and colistin resistance in Mecklenburg-Western Pomerania]. Epidemiologisches Bulletin. 2 October 2019. Available from: https://www.rki.de/DE/Content/Infekt/EpidBull/ Archiv/2019/40/Art_02.html

10. Abu Sin M, Blank H-P, Eckmanns T, Haller S. Die Bedeutung von Screeninguntersuchungen bei nosokomialen Ausbrüchen aus epidemiologischer Perspektive. Hyg Med. 2016;41:D32-7.

11. Friedrich AW, Daniels-Haardt I, Köck R, Verhoeven F, Mellmann A, Harmsen D, et al. EUREGIO MRSA-net Twente/Münsterland-a Dutch-German cross-border network for the prevention and control of infections caused by methicillin-resistant Staphylococcus aureus. Euro Surveill. 2008;13(35):13. https:// doi.org/10.2807/ese.13.35.18965-en PMID: 18761882

12. Guducuoglu H, Gursoy NC, Yakupogullari Y, Parlak M, Karasin G, Sunnetcioglu M, et al. Hospital Outbreak of a Colistin-Resistant, NDM-1- and OXA-48-Producing Klebsiella pneumoniae: High Mortality from Pandrug Resistance. Microb Drug Resist. 2018;24(7):966-72. https://doi.org/10.1089/ mdr.2017.0173 PMID: 29265963

13. Pirš M, Cerar Kišek T, Križan Hergouth V, Seme K, Mueller Premru M, Jeverica S, et al. Successful control of the first OXA-48 and/or NDM carbapenemase-producing Klebsiella pneumoniae outbreak in Slovenia 2014-2016. J Hosp Infect. 2019;101(2):142-9. https://doi.org/10.1016/j.jhin.2018.10.022 PMID: 30399389

14. Gona F, Bongiorno D, Aprile A, Corazza E, Pasqua B, Scuderi MG, et al. Emergence of two novel sequence types (3366 and 3367) NDM-1- and OXA-48-co-producing K. pneumoniae in Italy. Eur J Clin Microbiol Infect Dis. 2019;38(9):1687-91. https://doi. org/10.1007/s10096-019-03597-w PMID: 31165962

15. Seiffert SN, Marschall J, Perreten V, Carattoli A, Furrer $\mathrm{H}$, Endimiani A. Emergence of Klebsiella pneumoniae coproducing NDM-1, OXA-48, CTX-M-15, CMY-16, QnrA and ArmA in Switzerland. Int J Antimicrob Agents. 2014;44(3):260-2. https://doi.org/10.1016/j.ijantimicag.2014.05.008 PMID: 25123809

16. Xie L, Dou Y, Zhou K, Chen Y, Han L, Guo X, et al. Coexistence of blaOXA-48 and Truncated blaNDM-1 on Different Plasmids in a Klebsiella pneumoniae Isolate in China. Front Microbiol. 2017;8:133. https://doi.org/10.3389/fmicb.2017.00133 PMID: 28210248 
17. Wyres KL, Hawkey J, Hetland MAK, Fostervold A, Wick RR, Judd

LM, et al. Emergence and rapid global dissemination of CTX-M15-associated Klebsiella pneumoniae strain ST307. J Antimicrob Chemother. 2019;74(3):577-81. https://doi.org/10.1093/jac/ dky492 PMID: 30517666

\section{License, supplementary material and copyright}

This is an open-access article distributed under the terms of the Creative Commons Attribution (CC BY 4.0) Licence. You may share and adapt the material, but must give appropriate credit to the source, provide a link to the licence and indicate if changes were made.

Any supplementary material referenced in the article can be found in the online version.

This article is copyright of the authors or their affiliated institutions, 2019. 